\title{
Identified Facilitator Neurons L29 and L28 Are Excited by Cutaneous Stimuli Used in Dishabituation, Sensitization, and Classical Conditioning of Aplysia
}

\author{
Robert D. Hawkins and Samuel Schacher \\ Center for Neurobiology and Behavior, College of Physicians and Surgeons, Columbia University, and New York State \\ Psychiatric Institute, New York, New York 10032
}

Tactile or electrical stimulation of the skin can be used to produce dishabituation, sensitization, and classical conditioning of the gill- and siphon-withdrawal reflex in Aplysia. These behavioral effects are thought to involve presynaptic facilitation at the synapses from siphon sensory neurons to gill and siphon motor neurons. Facilitation of PSPs onto the motor neurons can also be produced by intracellular stimulation of single identified neurons in the abdominal ganglion, including L29 and L28. In this paper, we further characterize L29 and L28. First, we show that they are excited by cutaneous stimuli similar to those used to produce dishabituation, sensitization, and classical conditioning and may therefore participate in mediating those behavioral effects. The results are also consistent with a possible role of L29 and L28 in higher-order features of conditioning. Second, we show that 5-HT does not mimic some of the PSPs of L29, in agreement with previous evidence that L29 is not serotonergic. Third, we present 2 types of evidence that $L 29$ acts directly to produce facilitation of the sensory cells: (1) L29 comes into close contact with sensory cells in fluorescent double-labeling experiments, and (2) L29 produces facilltation of sensory cells in dissociated cell culture. Together with the results of the preceding paper (Mackey et al., 1989), these results indicate that facilitation of sensory cell synapses contributing to behavioral enhancement of the reflex can be produced by identified neurons that use 2 different transmitters: 5-HT (the transmitter of CB1) and the unknown transmitter of L29.

The Aplysia gill- and siphon-withdrawal reflex can be dishabituated or sensitized by tactile or electrical stimulation of the neck, mantle shelf, or tail (Pinsker et al., 1970; Carew et al., 1971, 1981; Mackey et al., 1988; Marcus et al., 1988; V. F. Castellucci and R. D. Hawkins, unpublished observations). These effects are thought to be due in part to presynaptic facilitation at the synapses from the siphon sensory neurons to gill and siphon motor neurons, which can be produced by tail shock,

Received Feb. 27, 1989; revised June 6, 1989; accepted June 12, 1989.

This work was supported by grants from the National Institutes of Health (MH26212 and GM32099). We thank D. Glanzman, L. Eliot, E. Kandel, and I. Kupfermann for their comments, $K$. Hilten and L. Katz for preparing the figures, and $H$. Ayers and $A$. Krawetz for typing the manuscript.

Correspondence should be addressed to Dr. Robert D. Hawkins, Center for Neurobiology and Behavior, College of Physicians and Surgeons, Columbia University, 722 West 168th Street, New York, NY 10032.

Copyright $(1989$ Society for Neuroscience $0270-6474 / 89 / 124236-10 \$ 02.00 / 0$ nerve stimulation, or 5-HT (Castellucci et al., 1970; Brunelli et al., 1976; Castellucci and Kandel, 1976; Klein and Kandel, 1978; Hawkins et al., 1983). Pharmacological evidence suggests that 5-HT plays an important role in the facilitation produced by tail shock (Glanzman et al., 1989), and identified serotonergic neurons in the cerebral ganglion, the CB1 cells, participate in mediating the facilitation (Mackey et al., 1989). Facilitation of PSPs onto the motor neurons can also be produced by intracellular stimulation of single identified neurons in the abdominal ganglion, including L29 and L28 (Hawkins et al., 1981b). Hawkins et al. (1981a) characterized L29 and L28 in terms of their position, size, color, spontaneous firing and PSPs, responses to nerve stimulation, and synaptic connections with other identified neurons. Since then, Frost (1987 and in preparation) and Hawkins (unpublished observations) have observed several additional synaptic connections of L29 and L28, which are listed in Table 1, along with the previously reported connections. L29 refers to a small group of neurons that act presynaptically, producing broadening of the action potential in the siphon sensory neurons (Hawkins, 1981b). The mechanism of facilitation by L28 has not been investigated.

These findings suggested that L29 and L28 participate in mediating some of the facilitation produced by tail shock. However, several important questions remained to be addressed: are L29 and L 28 excited by cutaneous stimuli that produce facilitation? Are they serotonergic? Do they act directly on the sensory neurons? In this paper, we further characterize the L29 and L28 neurons and show that they are excited by cutaneous stimuli

Table 1. Synaptic connections of L29 and L28

\begin{tabular}{llll} 
L29 & & L28 \\
\hline EPSP from LE & 1 & E-ILD from LE & 1 \\
Slow PSP to LE & 1 & EPSP to L7 & 1 \\
EPSP to LBS & 1 & IPSP from L10 & 1 \\
EPSP to LFS & 2,3 & Inhib to L10 & 1 \\
IPSP from L16 & 2,3 & E-ILD to L14 & 1 \\
IPSP from L21 & 1 & EPSP from L29 & 1 \\
EPSP to L28 & 1 & E-ILD from L32 & 3 \\
EPSP to L30 & 1 & & \\
IPSP from L30 & 1 & & \\
Coupled to L30 & 1 & & \\
EPSP to L32 & 2,3 &
\end{tabular}

$1=$ Hawkins et al., 1981a; 2 = Frost, 1987 and in preparation; $3=$ Hawkins, unpublished. 


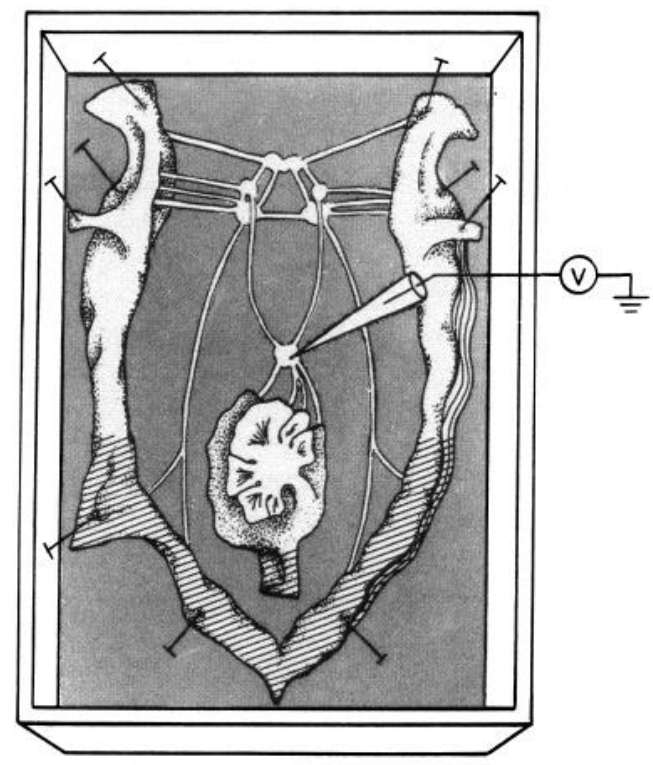

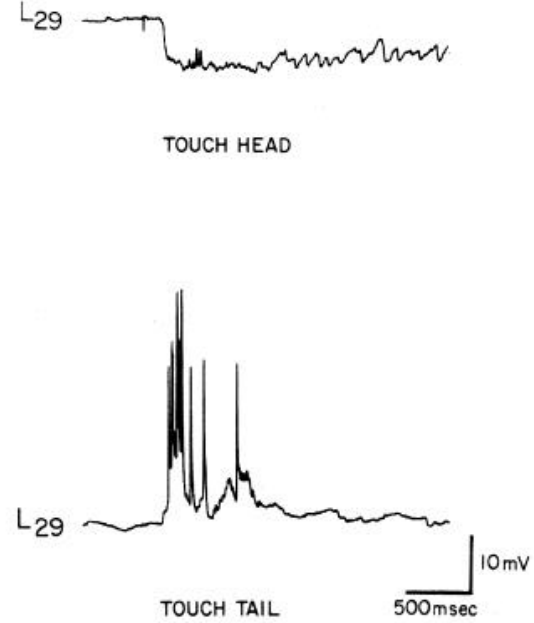

Figure 1. Cutaneous receptive field of L29. A, Shaded area indicates the excitatory receptive field of L29 in a semiintact preparation. $B$, Touching the head hyperpolarizes L29, whereas touching the tail produces a burst of spikes. similar to those used during dishabituation, sensitization, and classical conditioning of the gill- and siphon-withdrawal reflex. We also present additional evidence that L29 is not serotonergic, but that it does act directly to produce facilitation of the sensory cells.

\section{Materials and Methods}

Electrophysiology. Aplysia californica weighing 100-200 gm were obtained from Pacific Biomarine (Venice, CA) or Marinus (Long Beach, CA). Animals were anesthesized by injection of isotonic $\mathrm{MgCl}_{2}(50-$ $100 \%$ body weight) and were dissected in a $50 \%$ isotonic $\mathrm{MgCl}_{2}, 50 \%$ artificial seawater solution. In experiments examining the synaptic connections and morphology of neurons, the abdominal ganglion was dissected out, dipped in $0.5 \%$ glutaraldehyde for $45 \mathrm{sec}$ to kill muscle cells in the sheath, pinned to the Sylgard floor of a recording chamber, and partially desheathed. The preparation was then perfused with artificial seawater (in mM: $460 \mathrm{NaCl}, 10 \mathrm{KCl}, 55 \mathrm{MgCl}_{2}, 11 \mathrm{CaCl}_{2}, 10$ Tris, pH 7.6) at room temperature. The major nerves were wrapped around stimulation posts to facilitate identification of neurons. Neurons were impaled with single-barreled glass microelectrodes filled with $2.5 \mathrm{M} \mathrm{KCl}$ or $2 \mathrm{M}$ potassium citrate for recording and stimulation through a Wheatstone bridge circuit. Fast green was injected iontophoretically from microelectrodes filled with a $10 \%$ solution in water. 5-HT was applied extracellularly by iontophoresis from microelectrodes filled with 100 mM 5-HT oxalate.

In experiments examining responses to cutaneous stimulation, a modified "split-foot" preparation (Hening et al., 1979) was used. The viscera, buccal mass, opaline gland, and purple gland were excised, and the anterior half of the foot and back were bisected longitudinally, revealing the nervous system and blood vessels. The mantle organs were dissected free from the rest of the body except for their connections to the nervous system and circulatory system. In some experiments, the mantle organs or the anterior half of the body was removed. The preparation was then pinned dorsal side up to the wax floor of a lucite recording chamber filled with circulating, aerated artificial seawater. The cut end of the cephalic artery was cannulated and perfused with artificial seawater at room temperature. The abdominal ganglion was pinned on a Sylgard stage and partially desheathed. Cells were impaled with microelectrodes and examined for their responses to cutaneous tactile stimulation using a glass probe or electrical stimulation through fine silver wires, insulated except for a few millimeters at the tips, which were implanted in the skin.
Intracellular fluorescent labeling. Identified cells were iontophoretically injected with the fluorescent dye lissamine rhodamine B (LRB, Gurr, High Wycombe, England), or Lucifer yellow (Sigma). Injection electrodes were filled with a $5 \%$ solution of dye in water and had resistances approximately 5 times as high as comparable electrodes filled with $2.5 \mathrm{M} \mathrm{KCl}$. Dye was injected by passing $500 \mathrm{msec}$ hyperpolarizing pulses (1-2 nA) at $1 \mathrm{~Hz}$ for 5-90 min, depending on the size of the cell. The ganglion was then placed in a depression slide filled with artificial seawater or $2 / 3$ glycerol, $1 / 3$ sea water, and viewed on a Leitz epifluorescence microscope. LRB-injected cells exhibit red fluorescence with the N2 filter cube (excitation filter $530-560 \mathrm{~nm}$, mirror $580 \mathrm{~nm}$, barrier filter $580 \mathrm{~nm}$ ), and Lucifer-injected cells exhibit yellow fluorescence with the D filter cube (excitation filter $355-425 \mathrm{~nm}$, mirror $455 \mathrm{~nm}$, barrier filter $460 \mathrm{~nm}$ ). The two were photographed together by taking a double exposure on Kodak Ektachrome 400 film.

Dissociated cell culture. Previously published cell culture techniques were used (Schacher and Proshansky, 1983; Rayport and Schacher, 1986). In experiments without electrophysiological identification of cells, the ganglion was treated with protease for $2.5 \mathrm{hr}$ before desheathing and removal of the cells (Schacher and Proshansky, 1983). In experiments with electrophysiological identification of cells, the ganglion was partially desheathed and an identified cell was injected with fast green before treatment of the ganglion with protease. The protease treatment was reduced to $1.5 \mathrm{hr}$ to compensate for the increased exposure of the cells due to desheathing. The dye-injected cell was then removed and placed in culture. Standard electrophysiological techniques were used to test facilitation and spike broadening with cultured cells.

\section{Results}

\section{Response of L29 and L28 neurons to cutaneous stimulation} Tests of involvement in dishabituation and sensitization. Hawkins et al. (1981a) showed that L29 and L28 are excited both orthodromically and antidromically by stimulation of the pleural-abdominal connectives, which carry input from the neck and tail regions. However, they did not show that these neurons are excited by the cutaneous stimuli used to produce behavioral dishabituation and sensitization. To test the hypothesis that the L28 and L29 neurons participate in mediating the effects of cutaneous stimuli, we have recorded the activity of those neurons in the semiintact preparation. 
A
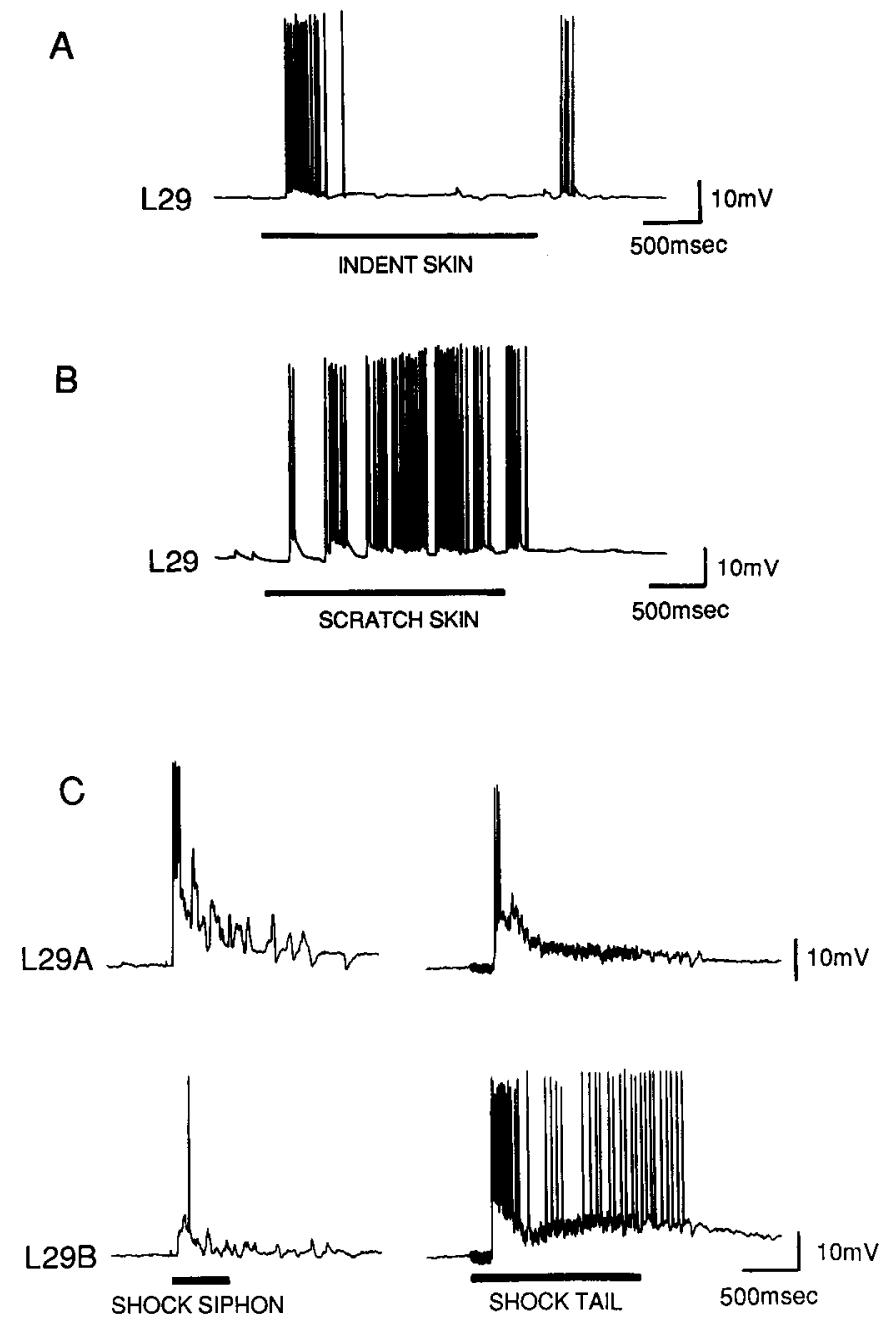

Figure 2. Response of $\mathrm{L} 29$ to cutaneous stimulation. A, Stationary indentation of the skin produces an "on" response and an "off" response in L29. B, Moving tactile stimulation (scratching) produces continuous firing of L29 during the stimulation. $C$, Simultaneously recorded responses of 2 L29 neurons to shock through electrodes implanted in the siphon $(5 \mathrm{~Hz}, 1.5 \mathrm{~V})$ and the tail $(10 \mathrm{~V}$ A.C. $)$.

The preparation we used is illustrated in Figure $1 A$. The animal was split longitudinally and pinned in a recording chamber, revealing the nervous system, so that we could record intracellularly from identified neurons while stimulating the skin. We first investigated the response of the L29 neurons. As shown in Figure $1 B$, touching the skin anywhere in the posterior part of the body produces a brisk burst of spikes in the L29 neurons. By contrast, touching the anterior part of the body produces hyperpolarization of the L29 cells. The shaded area in Figure $1 A$ indicates the excitatory receptive field of the $\mathrm{L} 29$ cells, which includes the tail, posterior parapodia and body wall, and siphon.

Within the excitatory region, stationary tactilc stimuli (indenting the skin) produce an adapting "on" response and also an "off" response (Fig. $2 A$ ), whereas moving tactile stimuli (scratching) produce sustained firing throughout the period of stimulation (Fig. $2 B$ ). As shown in Figure $2 C$, shocking the skin produces a high-frequency "on" response, which is often followed by a lower-frequency sustained response. The frequency of the "on" response can be greater than $50 \mathrm{~Hz}$, and the sustained response can be up to $40 \mathrm{~Hz}$. Sustained firing generally stops soon after the stimulation ends. Figure $2 C$ also shows that 2 different L29 cells in the same animal can have somewhat different receptive fields: in this experiment $\mathrm{L} 29_{\mathrm{A}}$ responded about equally to siphon and tail stimulation, whereas $\mathrm{L} 29_{\mathrm{B}}$ responded much more to the tail stimulation.

These results show that the L29 neurons are excited by cutaneous stimuli to the tail similar to those which have been used to produce dishabituation and sensitization of the gill- and siphon-withdrawal reflex. Figure 3 shows results from an experiment in which we recorded firing of L29 cells and behavioral dishabituation simultaneously in the semiintact preparation. The siphon was stimulated with an implanted electrode at 1-min intervals, producing excitation of L29 and a gill motor neuron (probably L7) and contraction of the gill which habituated with repeated stimulation (the response on trial 10 was approximately $10 \%$ of the response on trial 1 ). After trial 10 , the tail was scratched for several seconds, producing sustained irregular firing of L29 (a total of 174 spikes in $10 \mathrm{sec}$ ). Following the tail stimulation, siphon stimulation produced more spikes in the motor neuron and a much larger contraction of the gill (compare trials 9 and 10 with trials 11 and 12). These results show that cutaneous stimulation adequate to produce behavioral dishabituation (in this case, scratching the tail) also produces firing in L29 neurons. Moreover, the frequency and duration of L.29 firing are similar to those which have been used to produce presynaptic facilitation by intracellular stimulation of L29 (Hawkins et al., 1981b).

We also investigated the response of L28 to cutaneous stimulation. As shown in Figure $4 B$, touching either the tail or neck produces a brief burst of spikes in L28. Figure $5 B$ shows a similar response to shocking the siphon or tail. The shaded area in Figure $4 A$ indicates the excitatory receptive field of $\mathrm{L} 28$. Unlike L29, L28 responds to cutaneous stimulation anywhere on the body. Since L28 usually fires only a few spikes in response to touching or shocking the skin, however, it may not play a prominent role in mediating the facilitating effects of those stimuli.

Tests of involvement in classical conditioning. In addition to undergoing dishabituation and scnsitization, the gill- and siphon-withdrawal reflex can also undergo classical conditioning (Carew et al., 1981, 1983; Hawkins et al., 1986). In those experiments, the conditioned stimulus (CS) is tactile or electrical stimulation of the siphon, and the unconditioned stimulus (US) is electrical stimulation of the tail. Stimulation of either the siphon or the tail with parameters similar to those used in conditioning experiments produces firing of L29 neurons (Fig. 2C) and L28 (Fig. 5B). This finding suggests that these identified facilitator neurons could be involved in aspects of conditioning as well as dishabituation and sensitization.

Results of cellular experiments suggest that one mechanism of classical conditioning of the withdrawal reflex is activitydependent amplification of presynaptic facilitation of the sensory neurons (Hawkins et al., 1983; Walters and Byrne, 1983; Carew et al., 1984; Clark, 1984). However, these results do not exclude the possibility that other cellular mechanisms might also contribute (see Lukowiak, 1986; Colebrook and Lukowiak, 1988). One such possible mechanism is synergistic excitation of the facilitator neurons by the CS and US inputs, such that paired presentation of the CS and US produces more firing in those neurons (and hence more facilitation) than unpaired training. As a test of this idea, we recorded from L29 and L28 neurons while stimulating the siphon and tail either separately in time or temporally paired, as in conditioning. In the experiment shown 


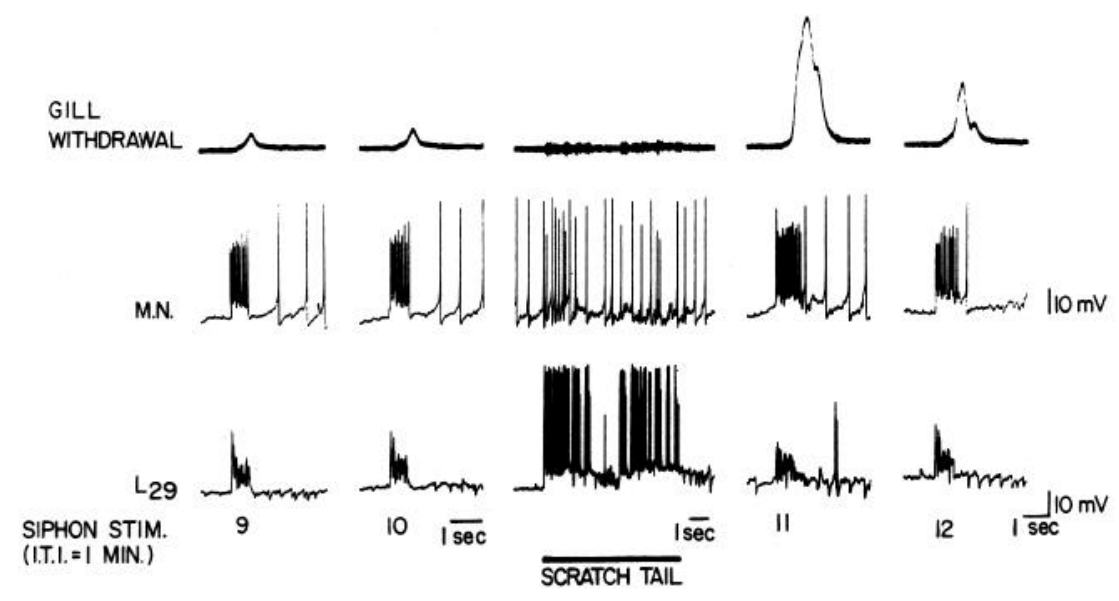

Figure 3. Response of $\mathrm{L} 29$ and a gill motor neuron during dishabituation of gill withdrawal. The siphon was stimulated through an implanted electrode $(6 \mathrm{~Hz}, 2.0 \mathrm{~V})$ once every $60 \mathrm{sec}$, producing depolarization in L29, firing in the motor neuron, and contraction of the gill (measured with a Grass tension transducer). Scratching the tail for several seconds between trials 10 and 11 produced a burst of spikes in L29 and an increase in the subsequent response of the motor neuron and the gill to stimulation of the siphon. in Figure $5 A$, we used moderate-intensity stimuli, which produced a few spikes in L29, for both the siphon and the tail. Contrary to the synergistic excitation hypothesis, siphon stimulation actually occluded tail stimulation; that is, paired stimulation produced no more spikes than siphon stimulation alone and fewer spikes than the total during unpaired stimulation. The occlusion illustrated in Figure $5 A$ is not due to habituation of the tail input, since tail stimulation alone produced the same number of spikes before and after the paired stimulation (not shown). It might be due in part to accommodation or recurrent inhibition of L29 (Hawkins et al., 1981a). With stronger stimulation the response to tail shock is not completely occluded by siphon stimulation, but paired stimulation still produces fewer spikes in L29 than unpaired stimulation. As shown in Figure $5 B$, paired stimulation also produces fewer spikes in L28 than unpaired stimulation. The occlusion in L28 may be due in part to the prominent afterhyperpolarization observed in that neuron.

\section{Additional evidence that L29 is not serotonergic: 5-HT does not mimic the EPSPs from L29 to L28 and L30}

Data from morphological studies and experiments on 5-HT uptake suggested that the L29 neurons might be serotonergic (Bailey et al., 1981, 1983). However, experiments with immunofluorescence and glyoxylic acid histofluorescence indicated that the L29 neurons do not contain 5-HT (Ono and McCaman, 1984; Kistler et al., 1985; Longley and Longley, 1986; Hawkins, 1989 b). As a further test of whether the L29 cells are serotonergic, we examined whether 5-HT mimics the conventional PSPs that L29 makes onto several follower cells. Serotonin was applied by iontophoresis either onto the cell body of the follower cell or into the neuropile between L29 and the follower; results

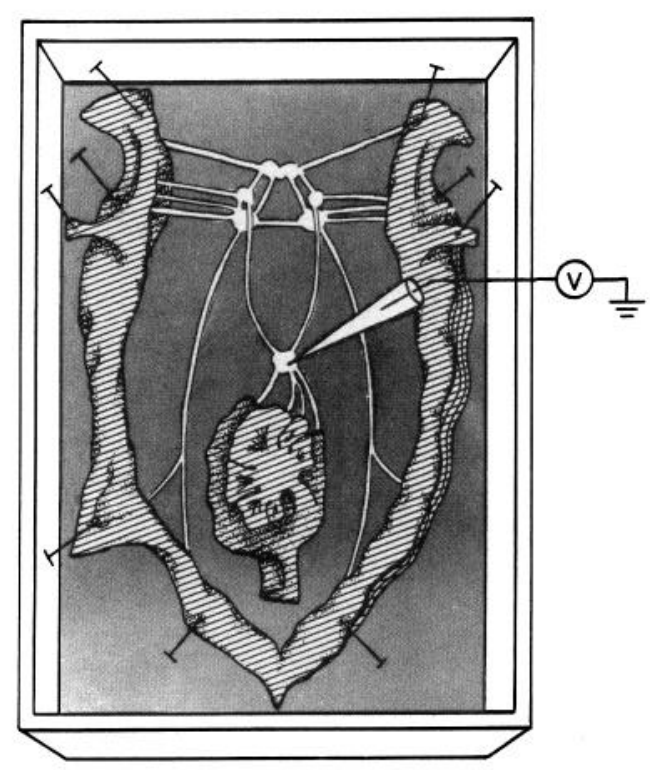

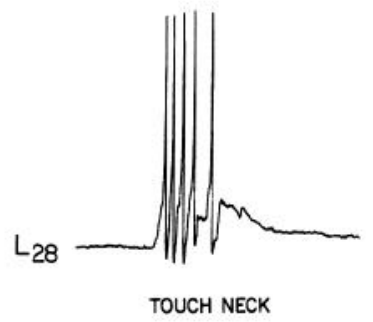

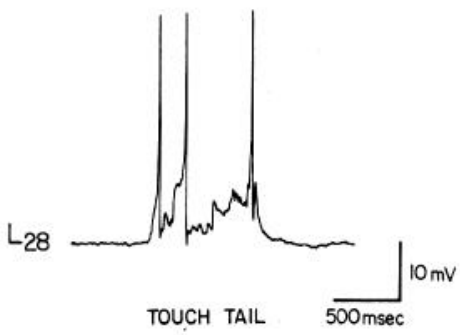

Figure 4. Response of $\mathrm{L} 28$ to cutaneous stimulation. $A$, Shaded area indicates the excitatory receptive field of L28. B, Touching the neck or tail produces a brief burst of spikes in L28. 


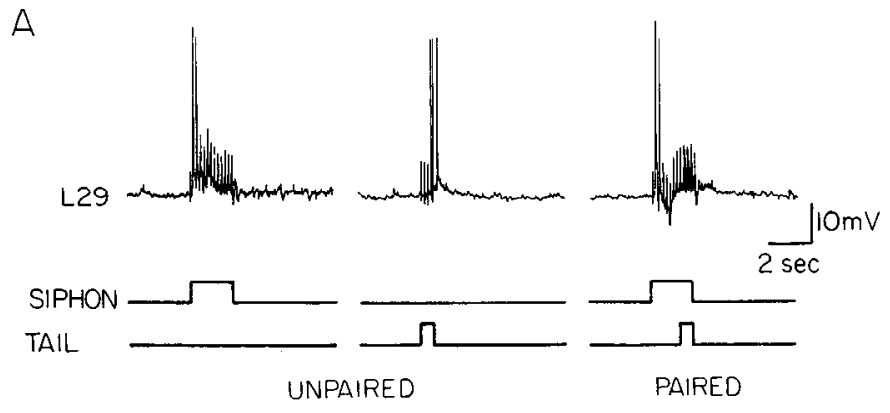

$\mathrm{B}$

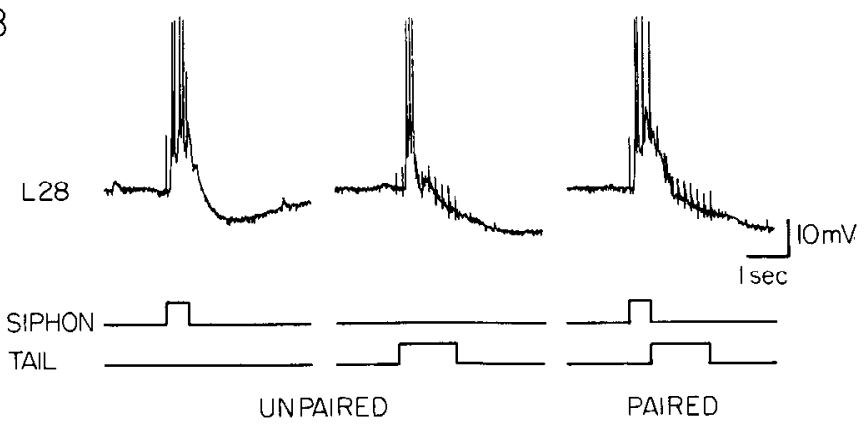

Figure 5. Response of L29 and L28 to unpaired and paired stimulation of the siphon and tail. $A$, Response of L29 to shock through electrodes implanted in the siphon $(6 \mathrm{~Hz}, 2.0 \mathrm{~V})$ and tail $(6 \mathrm{~Hz}, 12 \mathrm{~V})$. Paired stimulation of the siphon and tail produced no more spikes than stimulation of the siphon alone. $B$, Response of L28 to shock through electrodes implanted in the siphon $(6 \mathrm{~Hz}, 5 \mathrm{~V})$ and tail $(6 \mathrm{~Hz}, 50 \mathrm{~V})$. Paired stimulation produced no more spikes than stimulation of the siphon alone.

were qualitatively similar with the two methods. Responses were tested both in normal seawater and in high $\mathrm{Ca}^{2+}(60 \mathrm{~mm})$, high $\mathrm{Mg}^{2+}(140 \mathrm{mM})$ seawater, which raises the threshold of neurons and reduces indirect responses. The records shown are from experiments in high $\mathrm{Ca}^{2+}, \mathrm{Mg}^{2+}$ seawater.

We tested the LFS siphon motor neurons, L28, and L30. L29 produces a fast EPSP followed by a slow depolarization in LFS cells, which is mimicked by 5-HT application (unpublished data and Frost, 1987). However, 5-HT does not mimic the PSPs from L29 to either L28 or L30. L29 produces a fast EPSP in L28, whereas serotonin produces a slow hyperpolarization (Fig. $6 A$ ). Similarly, L29 facilitator neurons produce a fast EPSP followed by a slow depolarization in L30, whereas serotonin produces a slow hyperpolarization (Fig. $6 B$ ). The $\mathrm{L} 30$ response to serotonin also persists in low $\mathrm{Ca}^{2+}(1 \mathrm{mM})$, high $\mathrm{Mg}^{2+}(220$ $\mathrm{mM}$ ) seawater, which blocks all synaptic transmission (not shown), demonstrating that it is not the indirect result of stimulating other neurons.

The results of these experiments support the conclusion from the immunofluorescence and glyoxylic acid histofluorescence experiments that the L29 facilitator neurons are not serotonergic. In an effort to determine the transmitter of the L29 cells, we applied 24 different possible transmitters, including all of the conventional transmitters, SCP, and several 5-HT-related compounds, to the ganglion (by adding them to the bath) to see whether any of them would mimic the PSPs produced by L29. None of the compounds we tried produced depolarization of the L30 cells, suggesting that L29 may use a novel transmitter.
A
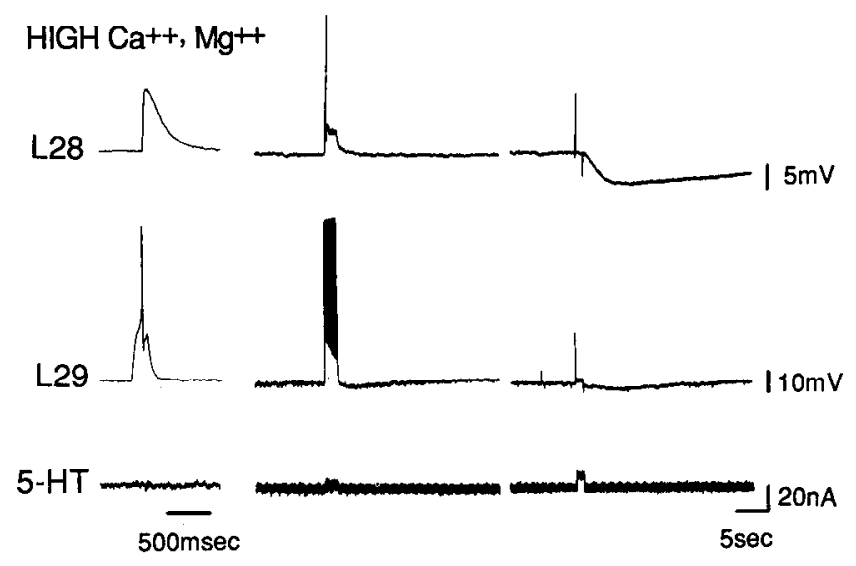

B
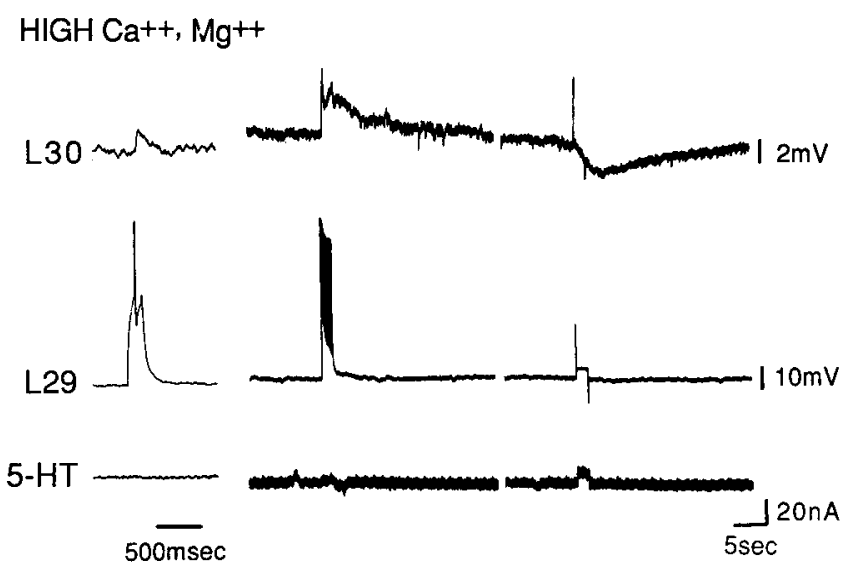

Figure 6. 5-HT does not mimic the EPSPs from L29 to L28 or L30. $A$, A single spike in L29 produces an EPSP in L28 in high $\mathrm{Ca}^{2+}(60$ $\mathrm{mM})$, high $\mathrm{Mg}^{2+}(140 \mathrm{mM})$ seawater, and a train of spikes produces a fast depolarization (note the change in time scale). Serotonin iontophoresis in the neuropile (current pulse recorded with a virtual ground) produces a slow hyperpolarization in L28. The virtual ground also recorded a small amount of current during intracellular stimulation of L29. $B$, A single spike in L29 produces an EPSP in L30 in high $\mathrm{Ca}^{2+}$, high $\mathrm{Mg}^{2+}$ seawater, and a train of spikes produces a fast and slow depolarization. 5-HT iontophoresis in the neuropile produces a slow hyperpolarization in $\mathrm{L} 30$.

\section{L29 acts directly on LE cells}

The finding that the L29 cells are not serotonergic raised the question of whether they produce facilitation of the siphon sensory cells directly, or whether they act indirectly by exciting other (perhaps serotonergic) facilitator neurons. Since the L29 cells do not produce a conventional PSP in the LE cells, the usual physiological tests of monosynapticity arc not suitablc. We have therefore taken 2 other approaches. The first is to examine the morphology of the L29 cells to see whether they come in close contact with the LE cells. The second is to test whether $\mathrm{L} 29$ cells can produce facilitation and spike broadening in LE cells in dissociated cell culture.

Light microscopic morphology of the L29 cells. To study the 


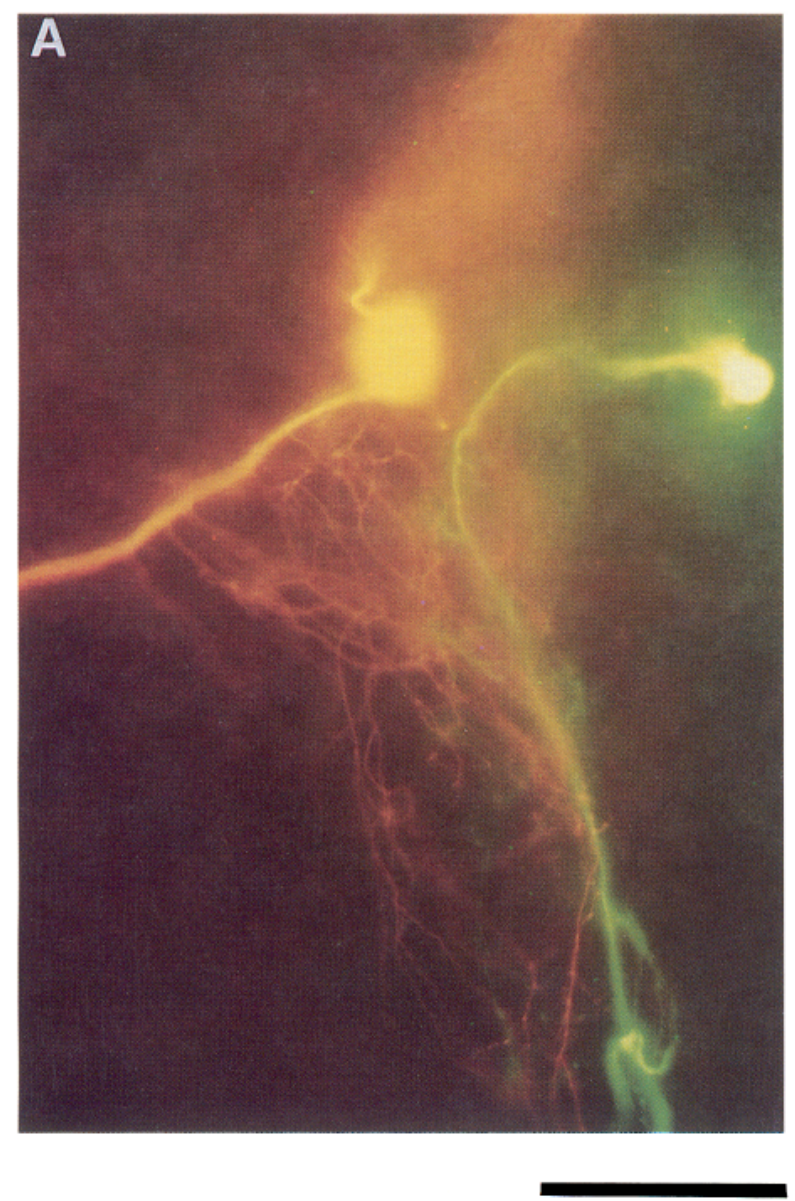

$250 \mu \mathrm{m}$

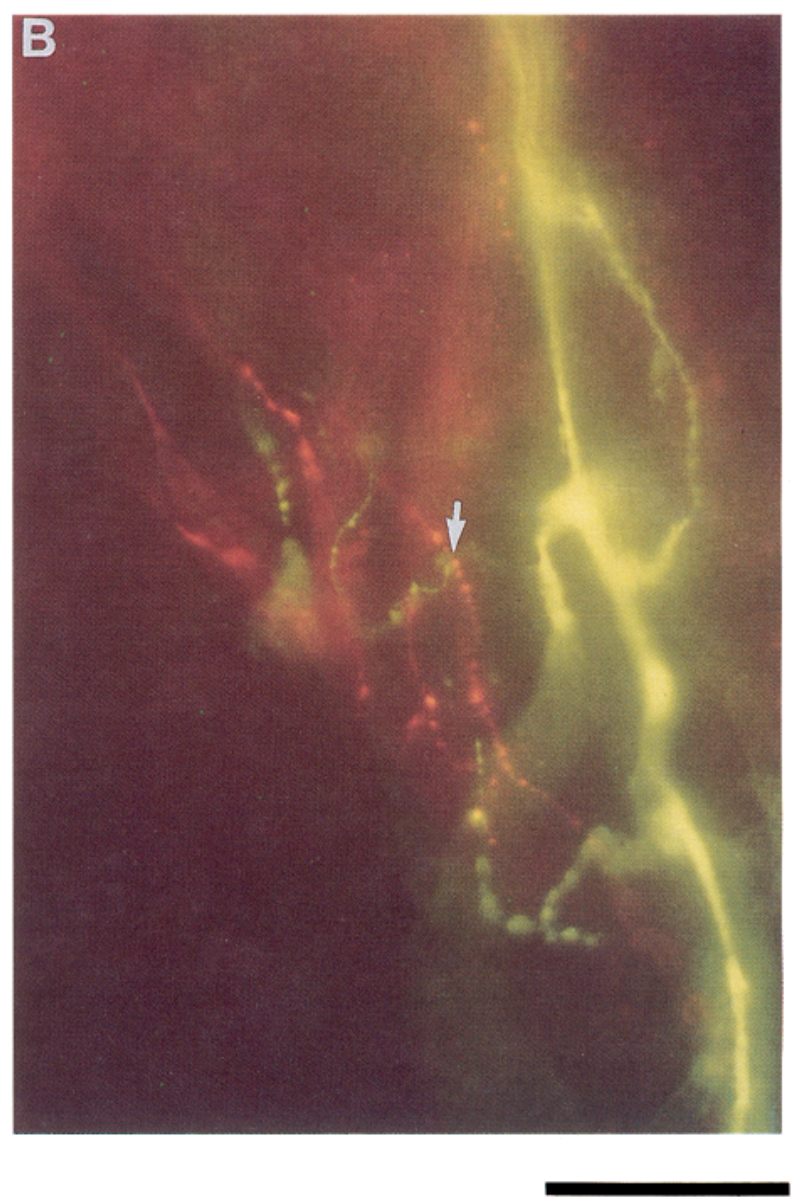

$100 \mu \mathrm{m}$

Figure 7. Intracellular fluorescent labeling of L29 and an LE sensory neuron. A, low-power view of the left ventral abdominal ganglion. An L29 facilitator neuron was injected with lissamine rhodamine B, which fluoresces red, and an LE sensory neuron was injected with Lucifer yellow, which fluoresces yellow-green. $B$, Higher-power view of the region in the lower-right-hand corner of $A$. The arrow points to an area where varicosities of the two neurons come in close contact.

morphology of the L29 cells, we injected a total of 22 L29 neurons with LRB, and in some experiments we also injected an LE sensory neuron with Lucifer yellow, or vice versa. The 2 dyes are approximately equally easy to inject and fill the fine processes of the cells comparably. Lucifer yellow seems to diffuse out the major axon more rapidly. As the 2 dyes have different fluorescent excitation and emission characteristics (rhodamine fluoresces red with the Leitz N2 filter cube and Lucifer yellow fluoresces yellow with the D filter cube), it is possible to identify unequivocally the fine processes of 2 different cells injected with the 2 dyes in the same ganglion.

Injected L29 cells invariably had 2 major axons: 1 entering the left pleural-abdominal connective, and the other crossing the abdominal commissure and entering the right pleural-abdominal connective. These results agree with reports of antidromic excitation of L29 by stimulating the left and right connectives (Hawkins et al., 1981a). However, an axon was never observed entering the branchial nerve, in disagreement with reports of occasional antidromic excitation of L29 by stimulation of that nerve (Hawkins et al., 1981a). Since judging antidromic excitation can be difficult, particularly if the site of spike initiation is far from the cell body, the dye filling results are probably more reliable. The L 29 cell body is usually monopolar, with the axon branching at some distance from it. Sometimes, however, the cell body is bipolar, with the 2 axons emerging from either end (Fig. 7A).

L29 cells have extensive branching of fine processes in 2 regions of the left abdominal ganglion: 1 posterior to the cell body and 1 anterior to it. There are also some fine processes in the right abdominal ganglion. The fine processes generally have a series of swellings that are presumed to be varicosities, or sites of transmitter release. In 1 experiment, 2 L29 neurons were injected in the same ganglion, 1 with rhodamine and the other with Lucifer yellow. The processes of the 2 cells occupied the same region of the ganglion but were not usually in contact with each other.

Figure 7 shows photographs of a ganglion in which an L29 facilitator cell was injected with lissamine rhodamine and an LE sensory cell was injected with Lucifer yellow. In agreement with previous reports (Bailey et al., 1979), the LE cell has one axon that enters the siphon nerve and a relatively sparse arbor of fine processes in the left hemiganglion (Fig. 7A). The processes of the 2 cells occupy the same region of the ganglion, and occasionally varicosities of the two cells come in close contact at the light microscopic level (Fig. $7 B$ ). These results indicate that L29 cells could act directly on the LE cells. 
A
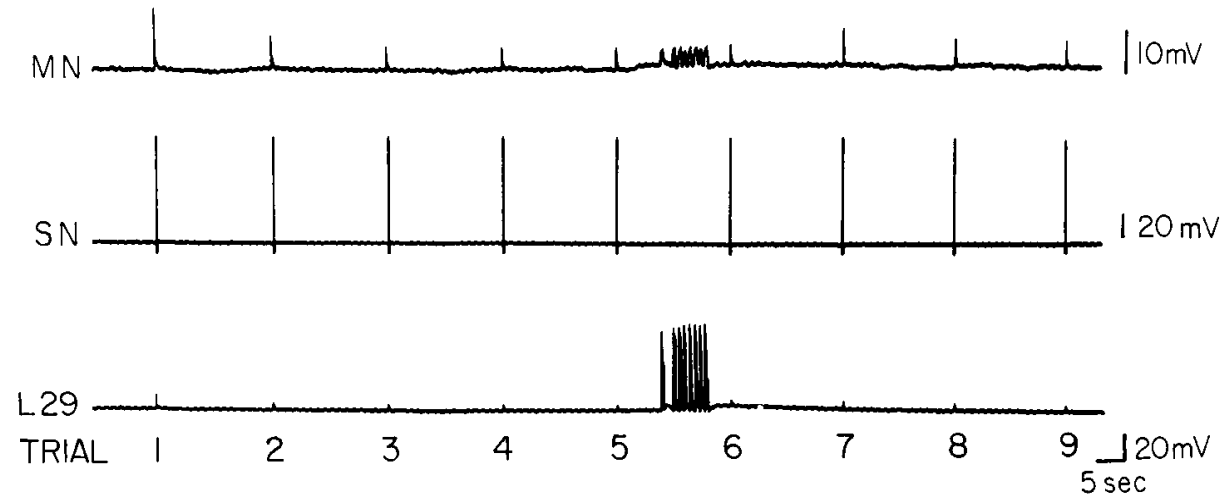

B

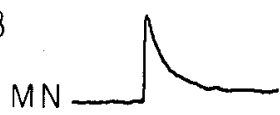

Figure 8. L29 produces facilitation of the EPSP from an LE sensory neuron to a motor neuron in dissociated cell culture. The LE neuron was stimulated intracellularly producing an EPSP in the motor neuron once every $20 \mathrm{sec}$. A train of spikes in L29 between trials 5 and 6 produced facilitation of the EPSP.
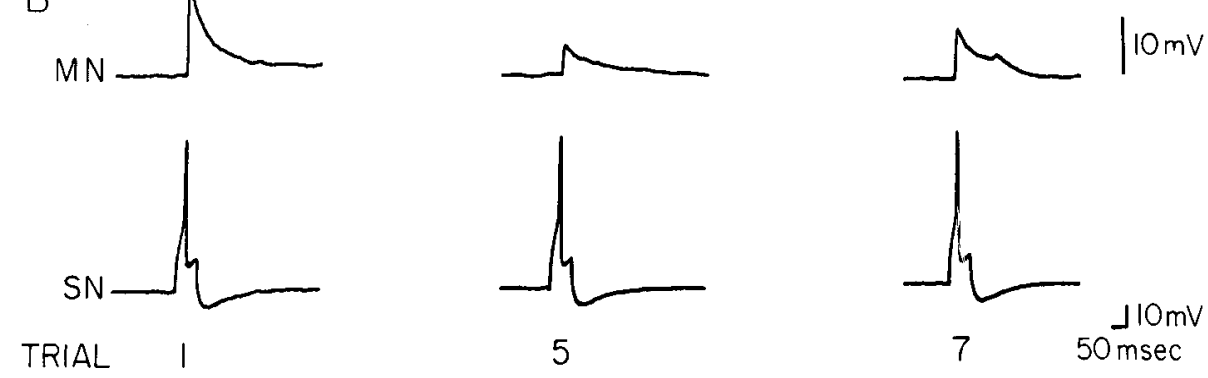

L29 neurons produce facilitation and spike broadening in dissociated cell culture. As a second test of whether L29 cells act directly on the LE cells, we examined whether they produce facilitation and spike broadening in LE cells in dissociated cell culture. Results from two successful experiments are shown in Figures 8 and 9.

In the experiment shown in Figure 8 , we identified a putative L29 neuron by its position in the ganglion, size, pigmentation, and axon projections (as determined when removing the cell from the ganglion after protease treatment), and cultured it with an LE neuron and 2 siphon motor neurons for 4 days. At that time the LE cell had formed excitatory chemical synaptic connections with the motor neurons and L29 (Fig. 8). The LE cell was then fired intracellularly once every $20 \mathrm{sec}$, producing an EPSP in 1 of the motor neurons that underwent homosynaptic depression (compare trials 1 and 5). Between trials 5 and 6, the L29 cell was stimulated intracellularly, causing it to fire a total of about 60 spikes in $8 \mathrm{sec}$. The EPSP from the LE cell to the motor cell was facilitated for the next several trials (compare trials 5 and 7). (The second bump on the EPSP on trial 7 is probably due to a spike in the nonrecorded motor neuron, which was weakly electrically coupled to the recorded motor neuron and L29.) Subsequent tests showed that L29 stimulation did not cause firing of either motor neuron, so the facilitation was not produced indirectly by exciting them. Interestingly, the onset of facilitation produced by L29 stimulation in this experiment was slightly more delayed than it is in vivo (Hawkins et al., 1981 b), suggesting that the L29 cell may not have made direct synaptic contact with the LE cell in vitro, but rather may have released its transmitter more diffusely.

In other experiments, we identified the L29 cell electrophysiologically (Hawkins et al., 1981a) and injected it with fast green before treating the ganglion with protease and removing the cell. In these experiments, we also tested whether the facilitation

produced by L29 in culture is presynaptic by measuring the duration of the action potential in the sensory neuron in tetraethylammonium (TEA) solution (Klein and Kandel, 1978; Hawkins, 1981b). In the experiment shown in Figure 9, the L29 cell and 3 LE cells were cultured together for 4 days. The cells were then perfused with $50 \mathrm{mM}$ TEA solution and 1 of the LE cells was fired with a brief intracellular current pulse once every $10 \mathrm{sec}$. Between trials 10 and 11 , the L29 cell was stimulated intracellularly, causing it to fire a burst of about 35 spikes in 5 sec. The action potential in the LE cell was broadened for approximately 2 min following the L29 stimulation (compare Pre and Post). This experiment was repeated while recording from each of the other LE cells in the dish, with similar results. As a control, firing a burst of spikes in one of the LE cells did not have any effect on the duration of the action potential in the other LE cells.

Stimulation of an identified L29 neuron produced broadening of the action potential in a sensory neuron in 2 of 5 cultures. We did not run enough of these technically difficult experiments for group statistics. However, as the incidence of spontaneous facilitation and spike broadening in culture is very low, it is highly unlikely that the results shown in Figures 8 and 9 are due to chance.

\section{Discussion}

Involvement of L29 and L28 in dishabituation, sensitization, and classical conditioning

Cutaneous stimuli to the tail that produce dishabituation and sensitization, such as scratching and shocking (Carew et al., 1981; Marcus et al., 1988; V. F. Castellucci and R. D. Hawkins, unpublished observations), cause firing of L29 neurons at a frequency and duration similar to those which have been used to produce presynaptic facilitation by intracellular stimulation 
A $50 \mathrm{mM}$ TEA

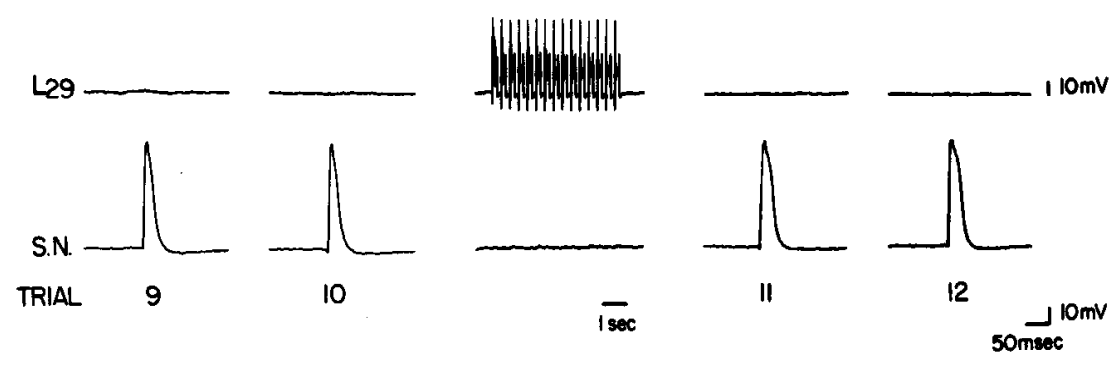

$B$

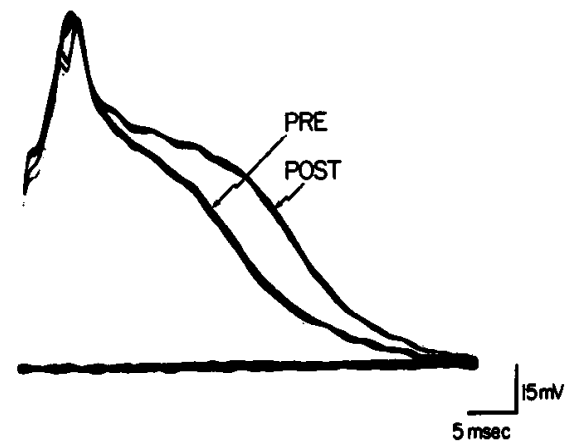

Figure 9. L29 produces broadening of the action potential in an LE sensory neuron in dissociated cell culture. The LE neuron was stimulated intracellularly once every $10 \mathrm{sec}$ in artificial seawater containing $50 \mathrm{mM}$ TEA. A train of spikes in L29 between trials 10 and 11 produced broadening of the action potential in the sensory neuron.

of L29 (Hawkins et al., 1981b). Brief reports of similar results have appeared previously (Hawkins, 1981a; Frost et al., 1988). These results strongly support the involvement of L29 neurons in mediating some of the facilitation contributing to dishabituation and sensitization. We have not yet tried the converse experiment of blocking facilitation, dishabituation, or sensitization by hyperpolarizing a single L29 neuron. However, removing a single neuron from the circuit might not be expected to reduce those effects very much, since many facilitator neurons probably contribute (Hawkins et al., 1981 b; Mackey et al., 1989).

Dishabituation and sensitization of the gill- and siphon-withdrawal reflex can be produced not only by tail stimulation, but also by stimulation of anterior body regions that do not excite L29 neurons (Pinsker et al., 1970; Carew et al., 1971; Mackey et al., 1988; Castellucci and Hawkins, unpublished observations), suggesting that other facilitator neurons mediate dishabituation and sensitization from those regions. We have shown that L28 is excited by stimulation of anterior as well as posterior body regions. In addition, Mackey et al. (1989) have identified a bilateral pair of serotonergic facilitator neurons in the cerebral ganglia, the CB1 cells, which are excited by stimulation anywhere on the body surface. The functional significance of this difference in receptive fields is not clear, but it suggests that L29 neurons have a relatively local role (see Mackey et al., 1989, for discussion of this point).

In addition to testing the involvement of L29 and L28 in dishabituation and sensitization, we have also examined their possible involvement in classical conditioning. Our results do not support 1 possible cellular mechanism of basic classical conditioning: synergistic excitation of these facilitator neurons by paired stimulation of the siphon and tail (the CS and US in conditioning experiments-Carew et al., 1981, 1983; Hawkins et al., 1986). Previous data suggest that conditioning is due instead to activity-dependent enhancement of facilitation of the sensory neurons (Hawkins et al., 1983; Walters and Byrne, 1983;
Carew et al., 1984; Clark, 1984). Specifically, tail shock (the US) produces presynaptic facilitation of all of the sensory neurons, but that facilitation is enhanced or amplified in those sensory neurons which are active just before the shock occurs. Since L29 and L28 are both excited by tail shock, they could mediate some of the facilitation contributing to basic classical conditioning.

Our results are also consistent with a possible role of L29 and L28 in higher-order features of conditioning. Hawkins and Kandel (1984) suggested that some of those features, including second-order conditioning and blocking, might be accounted for by the properties of the facilitator neurons illustrated in Figures $2 C$ and 5: (1) the facilitators are excited by both the CS and the US, and (2) firing of the facilitator neurons accommodates, so that the response to the CS actually tends to occlude the response to the US with paired stimulation. Computer simulations (Hawkins, 1989a) have demonstrated that these properties of the facilitators could contribute to second-order conditioning and blocking. To test these hypotheses physiologically, it will be necessary to record from the facilitator neurons simultaneously with behavioral conditioning, as we have done for L29 during dishabituation (Fig. 3). This may be feasible, as it is possible to produce conditioning in a semiintact preparation similar to the one we have used in these experiments (Lukowiak, 1986; Colebrook and Lukowiak, 1988; R. D. Hawkins, unpublished observations). Preliminary results from L29 in such experiments are consistent with the proposed mechanisms of second-order conditioning and blocking. It will also be important to record from the other identified facilitator neurons, including the CB1 neurons. The ideas we have proposed for higher-order features of conditioning (Hawkins and Kandel, 1984; Hawkins, 1989a) are based on the L29 cells, which respond to cutaneous stimulation with a phasic burst of firing. The CB1 cells respond with a tonic increase in firing which may be more suited for producing long-lasting sensitization. It is therefore possible that 
these different facilitator neurons are specialized for different roles in learning (see Mackey et al., 1989, for further discussion of this point).

\section{L29 neurons are not serotonergic}

Several previous lines of evidence suggested that the L29 cells might be serotonergic. First, 5-HT and stimulation of L29 cells both produce facilitation of the EPSPs from siphon sensory cells to follower cells, accompanied by a slow, decreased conductance EPSP in the sensory neurons, reduction of the outward current in the sensory neurons under voltage clamp, and broadening of the sensory neuron action potential in TEA solution (Brunelli et al., 1976; Klein and Kandel, 1978, 1980; Hawkins et al., $1981 \mathrm{~b}$; Hawkins, $1981 \mathrm{~b}$ and unpublished observations). Second, the facilitation produced by L29 stimulation is reversibly reduced by the 5-HT antagonist cinanserin (Hawkins et al., 1976). Third, the L29 cells resemble known serotonergic neurons in Aplysia [the metacerebral cells (MCCs)] in the size, electron density, and regional distribution of their vesicles, in making invaginating axosomatic contacts, and in packaging intrasomatically injected ${ }^{3} \mathrm{H}-5-\mathrm{HT}$ preferentially within end-stage lysosomes (Schwartz et al., 1979; Shkolnik and Schwartz, 1980; Bailey et al., 1981). Finally, the L29 cells show a specific, highaffinity uptake of ${ }^{3} \mathrm{H}-5-\mathrm{HT}$ from the bath (Bailey et al., 1983).

More recent evidence, however, suggests that the L29 cells are not serotonergic. Biochemical assays for 5-HT synthesis or presence in the L29 cells have not yielded positive results, although the small size of the cells makes a negative biochemical result inconclusive (J. H. Schwartz, R. E. McCaman, and R. D. Hawkins, unpublished observations). The L29 cells have also failed to show 5-HT immunoreactivity or glyoxylic acid histofluorescence in several studies (Ono and McCaman, 1984; Kistler et al., 1985; Longley and Longley, 1986; Hawkins, 1989b). Glanzman et al. (1989) found that the 5-HT neurotoxin 5,7DHT does not affect the mediating or modulating synaptic actions of L29, and the present study demonstrates that 5-HT application does not mimic the PSPs from L29 to its follower cells, L28 and L30. The negative results from these independent methods (immunofluorescence, glyoxylic acid histofluorescence, and electrophysiology), which are not limited by the size of the cells, strongly suggest that the L29 cells are not serotonergic. As none of the conventional transmitters we tried mimicked the L29 PSPs, L29 may use a novel transmitter.

Results with the immunofluorescence and glyoxylic acid methods also suggest that the L28 neurons are not serotonergic, as no serotonergic cells are seen in the region of the abdominal ganglion where L28 cells are usually located (Hawkins et al., 1981a; Kistler et al., 1985; Hawkins, 1989b). Electrophysiologically identified L28 cells have not yet been tested, however.

\section{$L 29$ acts directly to produce facilitation of $L E$ sensory neurons}

The conclusion that the L 29 cells are not serotonergic raises the question of whether they produce facilitation of the siphon sensory cells directly, or whether they act indirectly by exciting other facilitator neurons. The fact that $\mathrm{L} 29$ cells can produce facilitation in high $\mathrm{Ca}^{2+}$, high $\mathrm{Mg}^{2+}$ solution (Hawkins, 1981b) suggests that they act directly. However, to produce facilitation it is usually necessary to fire a train of spikes in the L29 neuron, which could excite interneurons even in high $\mathrm{Ca}^{2+}, \mathrm{Mg}^{2+}$ solution (see Fig. 6A). Moreover, in only about one-third of the experiments do L29 cells produce facilitation (Hawkins et al., $1981 \mathrm{~b}$ ). There are many possible interpretations of this result (see Hawkins et al., 1981b), but one is that L29 cells may act indirectly by exciting other neurons.

We have tested this possibility in 2 ways. First, we have shown that the L29 cells come in close contact with the LE sensory cells in double fluorescent-labeling experiments. This result is consistent with the electron microscopic observations of Bailey et al. (1981), and supports the possibility that the L29 cells may act directly on the LE cells. Second, we have shown that L29 stimulation produces facilitation and spike broadening in LE sensory cells in dissociated cell culture. Rayport and Schacher (1986) have previously shown that stimulation of the serotonergic MCCs produces facilitation of PSPs from LE sensory cells to motor cells in culture. Since the MCCs do not come into contact with the LE cells in vivo, however, this is a somewhat artificial situation. The present results are the first reconstruction of the natural circuit in vitro and demonstrate that the L29 cells release a facilitating transmitter. This conclusion is also supported by the results of Glanzman et al. (1984), who showed that extracts of L29 cells produce spike broadening in LE sensory cells. Coupled with the morphological data, these results strongly suggest that the L29 cells act directly to produce facilitation of the LE cells in vivo.

\section{Multiple facilitating transmitters}

The results of these experiments indicate that the L29 cells release an unknown transmitter that acts directly to produce facilitation at the synapses from siphon sensory neurons to motor neurons. Facilitation at those synapses can also be produced by application of 5-HT or stimulation of identified serotonergic neurons, the CBI cells (Brunelli et al., 1976; Mackey et al., 1989), and by application of an endogenous peptide, SCP (Abrams et al., 1984). It is not clear why there is more than one facilitating transmitter for the same synapses. Recent evidence indicates that 5-HT and SCP have somewhat different physiological effects at the sensory neuron synapses (Schacher and Montarolo, 1988). The identification of individual facilitator neurons (such as L29 and CB1), which use different transmitters, should make it possible to begin to study the functional significance to the animal of having multiple facilitating transmitters.

\section{References}

Abrams, T. W., V. F. Castellucci, J. S. Camardo, E. R. Kandel, and P. E. Lloyd (1984) Two endogenous neuropeptides modulate the gill and siphon withdrawal reflex in Aplysia by presynaptic facilitation involving cAMP-dependent closure of a serotonin-sensitive potassium channel. Proc. Natl. Acad. Sci. USA 81: 7956-7960.

Bailey, C. H., E. B. Thompson, V. F. Castellucci, and E. R. Kandel (1979) Ultrastructure of the synapses of sensory neurons that mediate the gill-withdrawal reflex in Aplysia. J. Neurocytol. 8: 415-444.

Bailey, C. H., R. D. Hawkins, M. C. Chen, and E. R. Kandel (1981) Interneurons involved in mediation and modulation of gill-withdrawal reflex in Aplysia. IV. Morphological basis of presynaptic facilitation. J. Neurophysiol. 45: 340-360.

Bailey, C. H., R. D. Hawkins, and M. Chen (1983) Uptake of (3H) serotonin in the abdominal ganglion of Aplysia californica: Further studies on the morphological and biochemical basis of presynaptic facilitation. Brain Res. 272: 71-81.

Brunelli, M., V. Castellucci, and E. R. Kandel (1976) Synaptic facilitation and behavioral sensitization in Aplysia: Possible role of serotonin and CAMP. Science 194: 1178-1181.

Carew, T. J., V. F. Castellucci, and E. R. Kandel (1971) An analysis of dishabituation and sensitization of the gill-withdrawal refiex in Aplysia. Int. J. Neurosci., 2: 79-98.

Carew, T. J., E. T. Walters, and E. R. Kandel (1981) Classical conditioning in a simple withdrawal reflex in Aplysia californica. J. Neurosci. $1: 1426-1437$. 
Carew, T. J., R. D. Hawkins, and E. R. Kandel (1983) Differential classical conditioning of a defensive withdrawal reflex in Aplysia californica. Science 219: 397-400.

Carew, T. J., R. D. Hawkins, T. W. Abrams, and E. R. Kandel (1984) A test of Hebb's postulate at identified synapses which mediate classical conditioning in Aplysia. J. Neurosci. 4: 1217-1224.

Castellucci, V., and E. R. Kandel (1976) Presynaptic facilitation as a mechanism for behavioral sensitization in Aplysia. Science 194: 11761178.

Castellucci, V., H. Pinsker, I. Kupfermann, and E. R. Kandel (1970) Neuronal mechanisms of habituation and dishabituation of the gillwithdrawal reflex in Aplysia. Science 167: 1745-1748.

Clark, G. A. (1984) A cellular mechanism for the temporal specificity of classical conditioning of the siphon-withdrawal response in Aplysia. Soc. Neurosci. Abstr. 10: 268.

Colebrook, E., and K. Lukowiak (1988) Learning by the Aplysia model system: Lack of correlation between gill and gill motor neuron responses. J. Exp. Biol. 135: 411-429.

Frost, W. N. (1987) Mechanisms contributing to short- and long-term sensitization in Aplysia. Ph.D. Dissertation, Columbia University, New York.

Frost, W. N., G. A. Clark, and E. R. Kandel (1988) Parallel processing of short-term memory for sensitization in Aplysia. J. Neurobiol. 19: 297-334.

Glanzman, D. L., T. W. Abrams, R. D. Hawkins, and E. R. Kandel (1984) Extracts of L29 interneurons produce spike-broadening in sensory neurons of Aplysia. Soc. Neurosci. Abstr. 10:510.

Glanzman, D. L., S. L. Mackey, R. D. Hawkins, A. Dyke, P. E. Lloyd, and E. R. Kandel (1989) Depletion of serotonin in the nervous system of Aplysia reduces the behavioral enhancement of gill withdrawal as well as the heterosynaptic facilitation produced by tail shock. J. Neurosci. 9: 4200-4213.

Hawkins, R. D. (1981a) Identified facilitating neurons are excited by cutaneous stimuli used in sensitization and classical conditioning of Aplysia. Soc. Neurosci. Abstr. 7: 354.

Hawkins, R. D. (1981b) Interneurons involved in mediation and modulation of gill-withdrawal reflex in Aplysia. III. Identified facilitating neurons increase $\mathrm{Ca}^{2+}$ current in sensory neurons. J. Neurophysiol 45: 327-339.

Hawkins, R. D. (1989a) A simple circuit model for higher-order features of classical conditioning, In Neural Models of Plasticity: Experimental and Theoretical Approaches, J. H. Byrne and W. O. Berry, eds., Academic, San Diego, CA.

Hawkins, R. D. (1989b) Localization of potential serotonergic facilitator neurons in Aplysia by glyoxylic acid histofluorescence combined with retrograde fluorescent labeling. J. Neurosci. 9: 4214-4226.

Hawkins, R. D., and E. R. Kandel (1984) Is there a cell biological alphabet for simple forms of learning? Psych. Rev. 91: 375-391.

Hawkins, R. D., V. F. Castellucci, and E. R. Kandel (1976) Identification of individual neurons mediating the heterosynaptic facilitation underlying behavioral sensitization in Aplysia. Soc. Neurosci. Abstr. 2: 325 .

Hawkins, R. D., V. F. Castellucci, and E. R. Kandel (1981a) Interneurons involved in mediation and modulation of gill-withdrawal reflex in Aplysia. I. Identification and characterization. J. Neurophysiol. 45: 304-314.

Hawkins, R. D., V. F. Castellucci, and E. R. Kandel (1981b) Interneurons involved in mediation and modulation of gill-withdrawal reflex in Aplysia. II. Identified neurons produce heterosynaptic facilitation contributing to behavioral sensitization. J. Neurophysiol. 45: 315-326.

Hawkins, R. D., T. W. Abrams, T. J. Carew, and E. R. Kandel (1983)
A cellular mechanism of classical conditioning in Aplysia: Activitydependent amplification of presynaptic facilitation. Science 219:400405.

Hawkins, R. D., T. J. Carew, and E. R. Kandel (1986) Effects of interstimulus interval and contigency on classical conditioning of the Aplysia siphon withdrawal reflex. J. Neurosci. 6: 1695-1701.

Hening, W. A., E. T. Walters, T. J. Carew, and E. R. Kandel (1979) Motorneuronal control of locomotion in Aplysia. Brain Res. 179: 231253.

Kistler, H. B., Jr., R. D. Hawkins, J. Koester, H. W. M. Steinbusch, E R. Kandel, and J. H. Schwartz (1985) Distribution of serotoninimmunoreactive cell bodies and processes in the abdominal ganglion of mature Aplysia. J. Neurosci. 5: 72-80.

Klein, M., and E. R. Kandel (1978) Presynaptic modulation of voltagedependent $\mathrm{Ca}^{2+}$ current: Mechanism for behavioral sensitization. Proc. Natl. Acad. Sci. USA 75: 3512-3516.

Klein, M., and E. R. Kandel (1980) Mechanism of calcium current modulation underlying presynaptic facilitation and behavioral sensitization in Aplysia. Proc. Natl. Acad. Sci. USA 77: 6912-6916.

Longley, R. D., and A. J. Longley (1986) Serotonin immunoreactivity of neurons in the gastropod Aplysia californica. J. Neurobiol. 17:339358.

Lukowiak, K. (1986) In vitro classical conditioning of a gill withdrawal reflex in Aplysia: Neural correlates and possible neural mechanisms. J. Neurobiol. 17: 83-101.

Mackey, S. L., N. Lalevic, R. D. Hawkins, and E. R. Kandel (1988) Comparison of dishabituation and sensitization of the gill-withdrawal reflex in Aplysia. Soc. Neurosci. Abstr. 14:842.

Mackey, S. L., E. R. Kandel, and R. D. Hawkins (1989) Identified serotonergic neurons LCBI and RCBI in the cerebral ganglia of $A p l y-$ sia produce presynaptic facilitation of siphon sensory neurons. J. Neurosci. 9: 4227-4235.

Marcus, E. A., T. G. Nolen, C. H. Rankin, and T. J. Carew (1988) Behavioral dissociation of dishabituation, sensitization, and inhibition in Aplysia. Science 241: 210-213.

Ono, J., and R. E. McCaman (1984) Immunocytochemical localization and direct assays of serotonin-containing neurons in Aplysia. Neuroscience 11: 549-560.

Pinsker, H., I. Kuperfmann, V. Castellucci, and E. R. Kandel (1970) Habituation and dishabituation of the gill-withdrawal reflex in Aplysia. Science 167: 1740-1742.

Rayport, S. G., and S. Schacher (1986) Synaptic plasticity in vitro: cell culture of identified Aplysia neurons mediating short-term habituation and sensitization. J. Neurosci. 6: 759-763.

Schacher, S., and P. G. Montarolo (1988) Serotonin selectively produces long-term facilitation of Aplysia sensorimotor synapses in culture. Soc. Neurosci. Abstr. 14: 909.

Schacher, S., and E. Proshansky (1983) Neurite regeneration by Aplysia neurons in dissociated cell culture: Modulation by Aplysia hemolymph and the presence of the initial axon segment. J. Neurosci. 3: 2403-2413.

Schwartz, J. H., L. J. Shkolnik, and D. J. Goldberg (1979) Specific association of neurotransmitters with somatic lysosomes in an identified serotonergic neuron of Aplysia californica. Proc. Natl. Acad. Sci. USA 76: 5967-5971.

Shkolnik, L. J., and J. H. Schwartz (1980) Genesis and maturation of serotonergic vesicles in identified giant cerebral neuron of Aplysia. J. Neurophysiol. 43: 945-967.

Walters, E. T., and J. H. Byrne (1983) Associative conditioning of single sensory neurons suggests a cellular mechanism for learning. Science 219: 405-408. 